\title{
Ambulatory Blood Pressure Monitoring in Diabetes and Obesity-A Review
}

\author{
Kazuo Eguchi \\ Division of Cardiovascular Medicine, Department of Medicine, School of Medicine, Jichi Medical University, \\ 3311-1 Yakushiji, Shimotsuke, Tochigi 329-0498, Japan \\ Correspondence should be addressed to Kazuo Eguchi, ke112@jichi.ac.jp
}

Received 11 January 2011; Accepted 20 January 2011

Academic Editor: Kazuko Masuo

Copyright ( $(2011$ Kazuo Eguchi. This is an open access article distributed under the Creative Commons Attribution License, which permits unrestricted use, distribution, and reproduction in any medium, provided the original work is properly cited.

Diabetes mellitus and obesity are both related to the risk of cardiovascular disease and sudden death. In hypertensive guidelines, diabetes and obesity, especially abdominal obesity, are regarded as high-risk factors. Ambulatory blood pressure monitoring $(\mathrm{ABPM})$ is an established method for the management of hypertension. However, ABPM is not a standard tool for the management of hypertension in diabetes and obesity. In this paper, recent data on the use of ABPM in diabetes and obesity will be discussed. In patients with diabetes, the ambulatory BP level has been shown to be better than clinic BP in predicting cardiovascular events. A riser pattern has been associated with increased risk of cardiovascular disease. White-coat hypertension and masked hypertension in diabetics constitute a moderate risk. A nondipping pattern is very common in obese hypertensive patients. In this paper, we will summarize the findings on the use of ABPM in patients with diabetes and obesity.

\section{Introduction}

There have been increasing numbers of diabetic and obese patients in recent years. Hypertension coexisting with diabetes and obesity has a major impact on cardiovascular prognosis. Patients with diabetes and obesity usually have other risk factors, such as dyslipidemia, sleep apnea syndrome, and metabolic syndrome. Strict control of BP has been recommended in these patients. The ACCORD trial proved that aggressive BP control has no such benefit on cardiovascular prognosis in patients with diabetes [1], but a new target level of BP in diabetes has not yet been established in response to these findings. Therefore, individualized control of BP is becoming more important in this postACCORD era. In this paper, we summarized the data on ABPM in diabetes and obesity.

\section{Ambulatory Blood Pressure Monitoring in Diabetes}

Diabetes itself is classified as a high-risk factor for cardiovascular disease, and when hypertension coexists with diabetes, not only is the cardiovascular risk magnified, but cardiovascular target organ damages such as silent cerebral infarcts (SCIs) and left ventricular hypertrophy (LVH) may progress. This is why the target level of blood pressure in diabetes is set as low as $130 / 80 \mathrm{mmHg}$. In a seminal paper by de la Sierra et al. based on findings from 42,947 patients included in the Spanish Society of Hypertension, ABPM registry has shown that diabetes was associated with nondipping status [2].

In clinical practice, it is sometimes very hard to identify the true blood pressure level when the BP variability is very large. In such cases, 24-hour BP monitoring (ABPM) is useful for the assessment of the actual blood pressure level and the prediction of cardiovascular prognosis. In patients with diabetes, the guidelines of the International Diabetes Federation (IDF) recommend that 24-hour ambulatory monitoring (ABPM) be used if so-called white coat hypertension is suspected, and it is limited in case of suspected white-coat hypertension [3]. On the other hand, there are no recommendations in regard to $\mathrm{ABPM}$ in the guidelines of the Japanese Society of Hypertension [4] or the American Diabetes Association [5]. This is largely because the data on the use of ABPM in diabetes, while gradually increasing (including in our own studies), currently remains insufficient. 


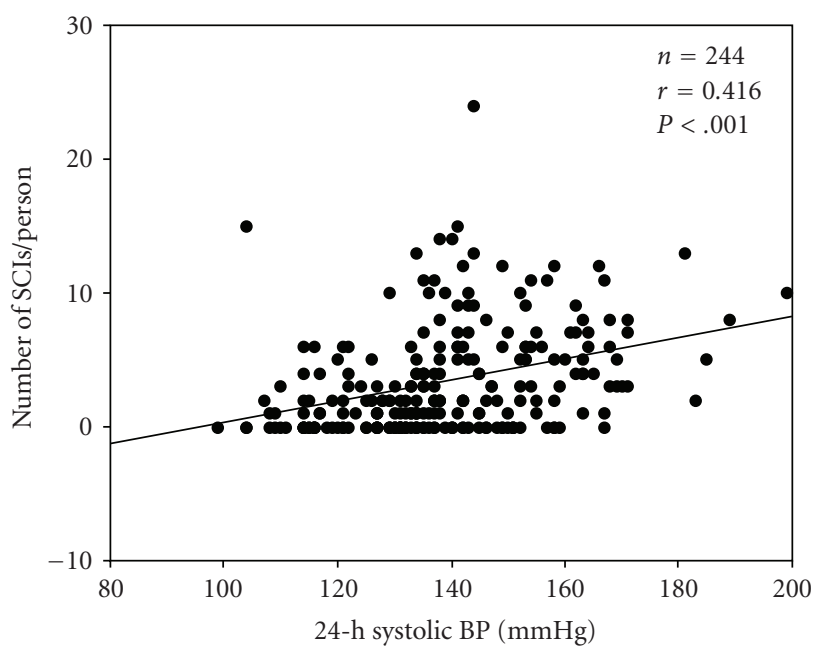

(a)

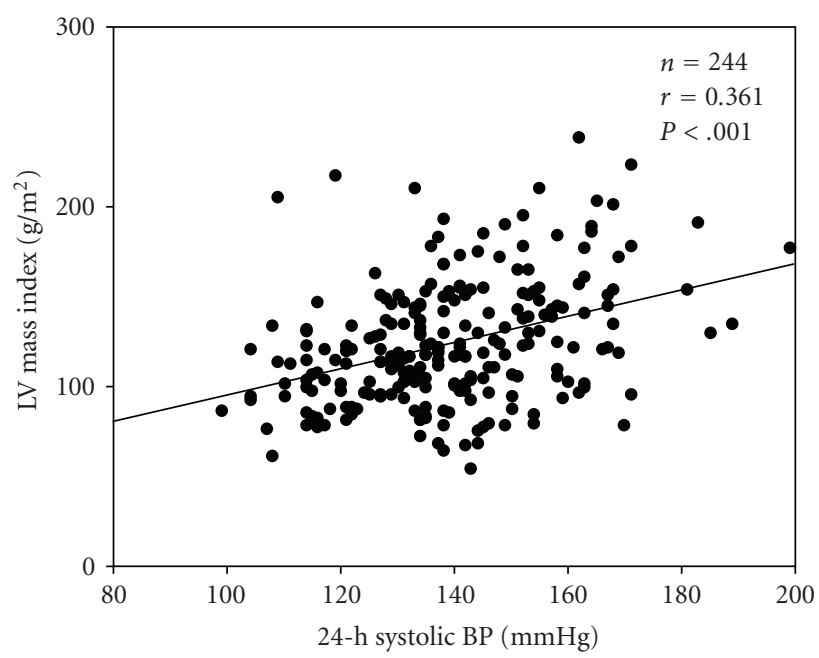

(c)

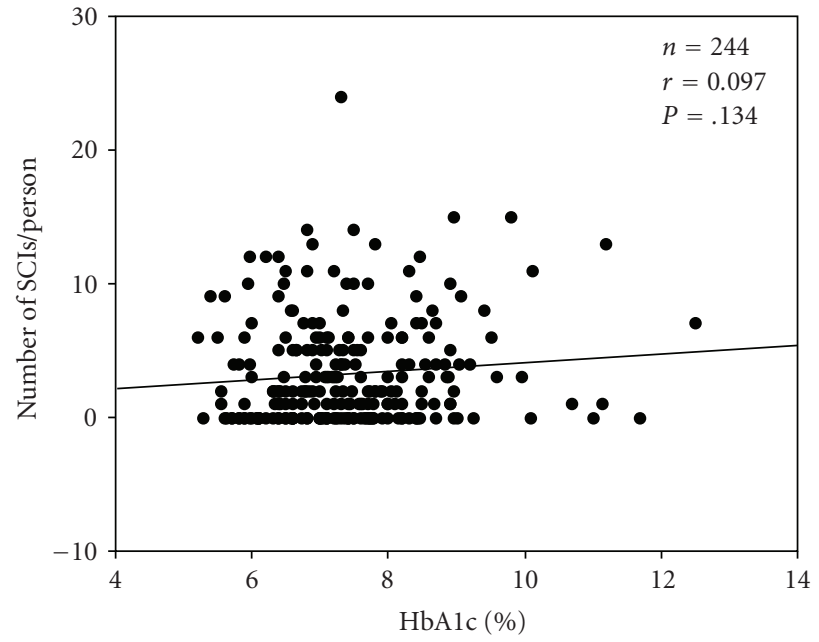

(b)

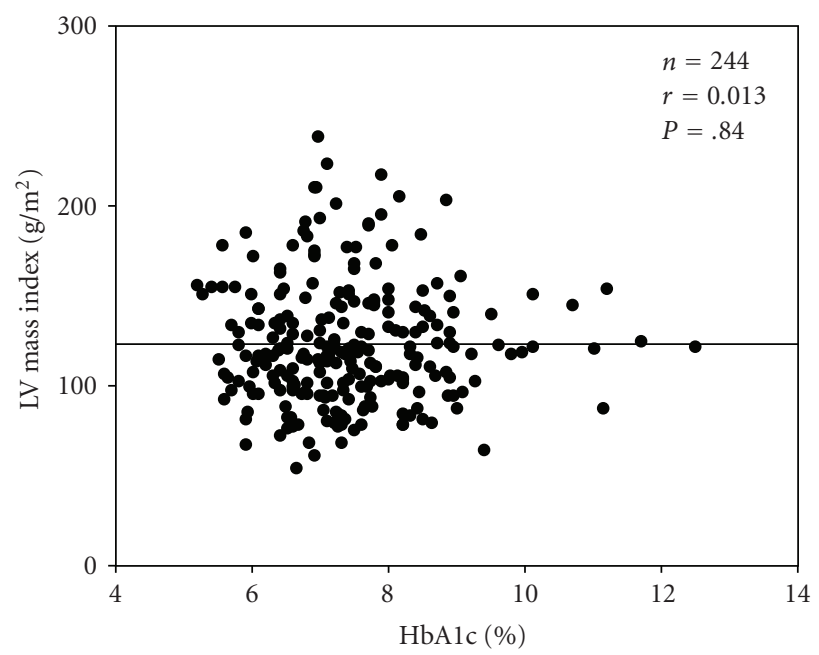

(d)

Figure 1: (a) and (b) represent 24-hour systolic blood pressure (BP), glycosylated hemoglobin (HbA1c), and number of silent cerebral infarcts (SCIs). (c) and (d) represent 24-hour systolic BP, HbAlc, and left ventricular (LV) mass index (adopted from [8]).

\section{Glucose Control and Ambulatory Blood Pressure Control: Which Is More Important in Preventing Cardiovascular Events in Diabetic Patients?}

Appropriate blood glucose control is a central part of the management of diabetes. Tight blood glucose control can lower not only microvascular complications, but also the risk of macrovascular disease. A prospective study in patients with type 2 diabetes reported an association between the degree of hyperglycemia and increased risk of stroke [6]. However, the United Kingdom Prospective Diabetes Study (UKPDS) reported that tight control of BP was more effective than tight control of blood glucose in the prevention of stroke $[7,8]$. Thus there has been some controversy about whether BP level or glycemic control is more strongly associated with future cerebrovascular events.
At present, there are few reports comparing the effects of ambulatory BP and glycemic factors on target organ damage in diabetic patients. We performed a cross-sectional analysis to investigate factors associated with target organ damage (SCI, LVH, and albuminuria) in 244 type 2 diabetics [9]. The mean age was 65.4 years, and the study group included 122 men and 122 women. As shown in Figures 1(a) and 1(b), 24-h systolic BP was significantly correlated with the number of SCIs $(r=0.416, P<.001)$, but the average hemoglobin A1c level was not $(r=0.097, P=.134)$. Similarly, as shown in Figures 1(c) and 1(d), 24-h systolic BP was positively correlated with the LV mass index $(r=0.361$, $P<.001$ ), but the hemoglobin Alc level was not positively correlated with the LV mass index $(r=0.013, P=.84)$. Then the subjects were divided into 4 groups according to the median values of ambulatory BP and hemoglobin A1c. As shown in Figure 2, the prevalence of SCI, multiple SCI and 


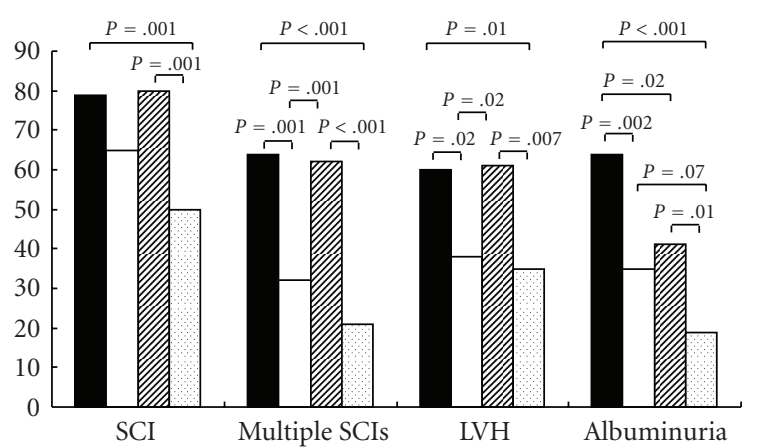

(\%)

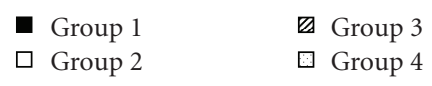

(a)

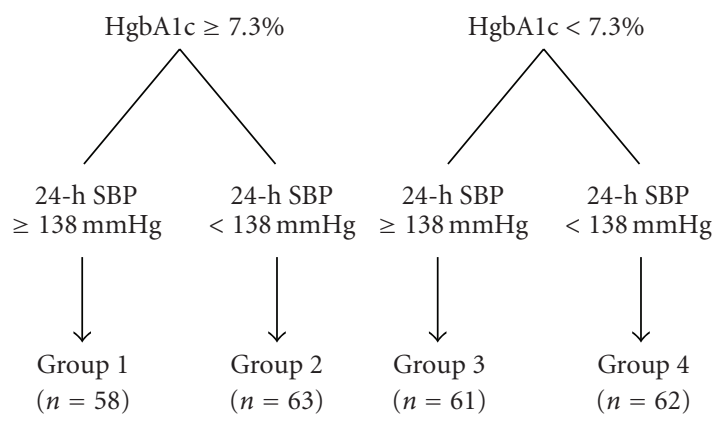

(b)

Figure 2: (a) Prevalence of hypertensive target organ damage in each group. SCI $=$ silent cerebral infarct; multiple SCIs $=$ three or more lesions; $\mathrm{LVH}=$ left ventricular hypertrophy; $P$-values noted above bars (adopted from [8]). (b) Classification of the subjects based on the median values of ambulatory systolic blood pressure (SBP) and glycosylated hemoglobin (HbAlc).

LVH were higher in Groups 1 and 3 (with high ambulatory BP) than in Group 2 (high HbAlc and normal 24-h BP) or 4 (normal HbA1c and normal 24-h BP). Group 1 had the highest value of albuminuria among the four groups. In multiple regression analyses, 24 -h systolic BP, age, and duration of hypertension were significantly correlated with the number of SCIs. On the other hand, 24-h SBP and male sex were significantly correlated with LVMI. We performed the same analysis for albuminuria, and found that 24-h systolic BP was most strongly correlated with the presence of albuminuria. Ambulatory BP control was shown to be more closely associated with target organ damage of the brain, heart, and kidney than glycemic control, and it is possible that ambulatory BP control is effective in preventing future risk of cardiovascular disease in patients with diabetes.

\section{Ambulatory Blood Pressure More Useful Than Clinic BP for the Management of Diabetic Patients?}

The effectiveness of ABPM in the management of hypertension in diabeticpatients has not been conclusively estab- lished. Nonetheless, we have shown that ambulatory BP was superior to clinic BP in predicting cardiovascular prognosis in patients with diabetes [10] (Figure 3). This finding was in agreement with a previous report by Nakano et al. showing that increased 24-hour pulse pressure was significantly associated with cardiovascular events in diabetes [11]. In combination with the findings of masked hypertension in diabetes (described below) [12], these results lead us to advocate that all diabetic subjects should undergo ABPM at least once regardless of the presence of hypertension. Even in cases of resistant hypertension in diabetes, pseudoresistance, that is, apparent hypertension in the clinic but normal BP outside the clinic, is sometimes observed due to the whitecoat effect [13].

\section{Is the Circadian BP Rhythm Useful in Predicting Cardiovascular Prognosis in Patients with Diabetes?}

In diabetes, an abnormal BP rhythm, that is, a non-dipper pattern, is frequently observed [2]. In patients with diabetes, nondipping pattern detected by a single ABPM study could be more reliable than nondiabetic patients as shown by Cuspidi et al. [14]. There have been several papers on circadian BP rhythm and CV prognosis in patients with diabetes. Nakano et al. performed ABPM in 325 patients with type 2 diabetes who they followed for 4 years to look at the effect of the riser pattern, an abnormal BP rhythm in which the average nighttime $\mathrm{BP}$ exceeds the average daytime BP, on future CV events [15]. Circadian BP rhythm was assessed by the COSINOR (an acronym formed from cosine and vector) method. A total of 288 patients were successfully followed, consisting of 201 dippers ( $N$ group) and 87 risers ( $R$ group). There were no differences in baseline gender, HbA1c, rate of smokers, lipids, or electrolytes, but the age, rate of hypertension, and rate of diabetic complications were higher in the $R$ group than in the $N$ group. During the follow-up period, fatal and nonfatal $\mathrm{CV}$ events (cerebrovascular, cardiovascular, peripheral vascular, and renovascular) occurred in 20 cases of the $N$ group and 56 cases of the $R$ group. Unadjusted survival and event-free survival were evaluated by Kaplan-Meier methods, and the $R$ group had a significantly higher number of events than the $N$ group (Log-rank $P<.001$, Figure 4 ). In multivariable analyses, after adjusting for various covariates, a riser pattern and age were significant predictors for both fatal and nonfatal events. Therefore, in type $2 \mathrm{DM}$, a riser pattern has been shown to be associated with fatal and nonfatal vascular events. In agreement with these results, we found that a riser pattern was associated with a $150 \%$ increase in the risk of $\mathrm{CV}$ disease even after adjusting for other covariates based on our data of diabetes and CV prognosis [10]. Therefore, for the prevention of CV disease in the context of diabetes, physicians should recognize that patients are at high risk when an abnormal circadian rhythm of BP is observed.

Lurbe et al. studied 75 young patients with type 1 diabetes who had had a normal BP for more than 5 years and were free from albuminuria at baseline. ABPM was 
TABle 1: $P$-values for the comparisons of 4 groups in Figure 6.

\begin{tabular}{lcc}
\hline & SCI & Multiple SCI \\
\hline WCHT versus SHT & 0.122 & 0.010 \\
WCHT versus WCHT + DM & 0.160 & 0.047 \\
WCHT versus SHT + DM & $<0.001$ & $<0.001$ \\
SHT versus WCHT + DM & 0.856 & 1.000 \\
SHT versus SHT + DM & $<0.001$ & $<0.001$ \\
WCHT + DM versus SHT + DM & 0.001 & 0.001 \\
\hline
\end{tabular}

WCHT $=$ white coat hypertension; $\mathrm{SHT}=$ sustained hypertension; $\mathrm{WCHT}+$ $\mathrm{DM}=$ white coat hypertension + diabetes mellitus; $\mathrm{SHT}+\mathrm{DM}=$ sustained hypertension + diabetes mellitus. $P$-values for SCI (multiple SCI) were calculated by chi-square test.

performed at baseline and 2 years later and the status of albuminuria was assessed [16]. Fourteen patients developed albuminuria, while the remaining 61 subjects did not. In the patients who developed albuminuria, nocturnal BP increased from $109.9 \pm 11.3$ to $114.9 \pm 11.7 \mathrm{mmHg}(P=$ $.01)$, but in patients who did not develop albuminuria, nocturnal BP did not change $(106.0 \pm 8.8$ versus $106.4 \pm$ $14.8 \mathrm{mmHg}$ ). The negative predictive value for the progression for albuminuria was $91 \%$ when the night/day ratio of $\mathrm{BP}$ was less than 0.9. Patients with a night/day ratio of $\mathrm{BP} \leq 0.9$ showed a $70 \%$ reduction of albuminuria $(95 \%$ CI $44-110, P=.01$, Figure 5) compared to those with a ratio $>0.9$. Therefore, even in normotensive diabetes, the importance of a nondipping pattern was confirmed in this study.

\section{White-Coat Hypertension in Diabetes}

The effects of white-coat hypertension in diabetes have not been fully studied. We performed ABPM and brain MRI in 360 patients with hypertension, diabetes, or both. SCI, a risk factor for future stroke, was evaluated by brain MRI [17]. Among the four groups, SCI was most frequently seen in diabetic patients with sustained hypertension (defined as both high clinic BP and high ambulatory BP) and was least frequently observed in nondiabetic patients with white-coat hypertension. On the other hand, the frequency of SCI was similar between nondiabetic patients with sustained HT and diabetic patients with WCH (Figure 6) Table 1 [18]. Whitecoat hypertension has been considered a low-risk factor for CV events; however, when coexistent with diabetes, whitecoat hypertension does have an impact via so-called whitecoat syndrome [19].

In our follow-up study on the above-described cohort, the CV prognosis of diabetic WCH was much better than in the diabetic SHT group (Figure 7) [20]. However, because the number of normotensive diabetics was relatively small, we could not compare the risk of $\mathrm{CV}$ events between diabetic WCH and diabetic normotensives. On the other hand, Kramer et al. performed a cross-sectional study which investigated the effect of white-coat HT in diabetes on the impact of microvessel disease [21]. They studied 319 type 2 diabetics who were normotensive or who had white-coat hypertension. Normotension was defined as office BP < $140 / 90 \mathrm{mmHg}$ and average daytime $\mathrm{BP}<135 / 85 \mathrm{mmHg}$, and white-coat HT as office $\mathrm{BP} \geq 140 / 90 \mathrm{mmHg}$ and average daytime BP $<135 / 85 \mathrm{mmHg}$. Diabetic nephropathy (DN: defined by 24-hour urinary albumin excretion) and diabetic retinopathy (DR: classification of the Global Diabetic Retinopathy Group) were assessed. The results showed that 46 patients with type $2 \mathrm{DM}$ were classified as having whitecoat hypertension $(14.4 \%)$, and 117 subjects were defined as normotensive (36.6\%). 24-hour SBP was higher in the $\mathrm{WCH}$ group than in the normotensive group (average 24hour BP: $124.7 \pm 6.7$ versus $121.0 \pm 8.5 \mathrm{mmHg}, P=.01$; average daytime BP: $126.6 \pm 7.2$ versus $123.2 \pm 8.2 \mathrm{mmHg}$, $P=.01)$. Of note, $\mathrm{WCH}$ was associated with the risk of overt albuminuria (odds ratio: $4.9,95 \%$ CI 1.3-18.7, $P=.01$ ). In the multivariable analysis, $\mathrm{WCH}$ was associated with overt albuminuria (odds ratio: 2.0; 95\% CI 1.3-3.2, $P=.02$ ) and nonproliferative and proliferative retinopathy (odds ratio, $2.7,95 \%$ CI 1.2-6.6, $P=.02$ ). The authors concluded that WCH with type $2 \mathrm{DM}$ was associated with an increased risk of diabetic retinopathy and nephropathy. Therefore, the combination of WCH and type 2 DM constitutes some level of risk for $\mathrm{CV}$ events and may require some treatment.

\section{Masked HT in DM}

Masked hypertension (MHT), a state office BP is normal range, but out-of-office $\mathrm{BP}$ is high and has been shown to be associated with future risk of $\mathrm{CV}$ events. However, the prevalence and clinical significance of MHT in patients with DM have not been fully investigated. We assessed the association of MHT (defined as a clinic BP $<140 / 90 \mathrm{mmHg}$ and daytime ambulatory BP $>135 / 85 \mathrm{mmHg}$ ) with microand macrovascular end organ damage in 81 clinically normotensive Japanese diabetics [12]. The prevalence of SCI and albuminuria was evaluated, and the left ventricular mass (LVM) was determined. Among the 81 patients, the 38 (46.9\%) who were classified as having MHT showed significantly more SCIs (mean \pm SEM: $2.5 \pm 0.5$ versus $1.1 \pm 0.2$, $P=.017$ ), and significantly higher incidence of albuminuria (39\% versus $16 \%, P=.025$ ), but no increase of LVMI compared to the true normotensive group. We concluded that the prevalence of MHT in this diabetic population was $47 \%$, a surprisingly high result. Diabetic patients with MHT showed evidence of brain and kidney damage. Leitão et al. performed a similar study in 135 normotensive patients with type 2 diabetes [22]. Patients underwent urinary albumin excretion rate (UAER) measurement, echocardiography, and 24-h ambulatory blood pressure monitoring (ABPM). The definition of masked hypertension was the same as in our study. The prevalence of masked hypertension was 30\% $(n=41)$. UAER and LV wall thickness were significantly higher in the group with masked hypertension than in the normotensives. After adjustments for covariates, all associations were sustained for daytime systolic blood pressure but not for office systolic blood pressure. Hence out-of-office monitoring of BP may be indicated even in diabetics whose $\mathrm{BPs}$ are normal in the clinic. 


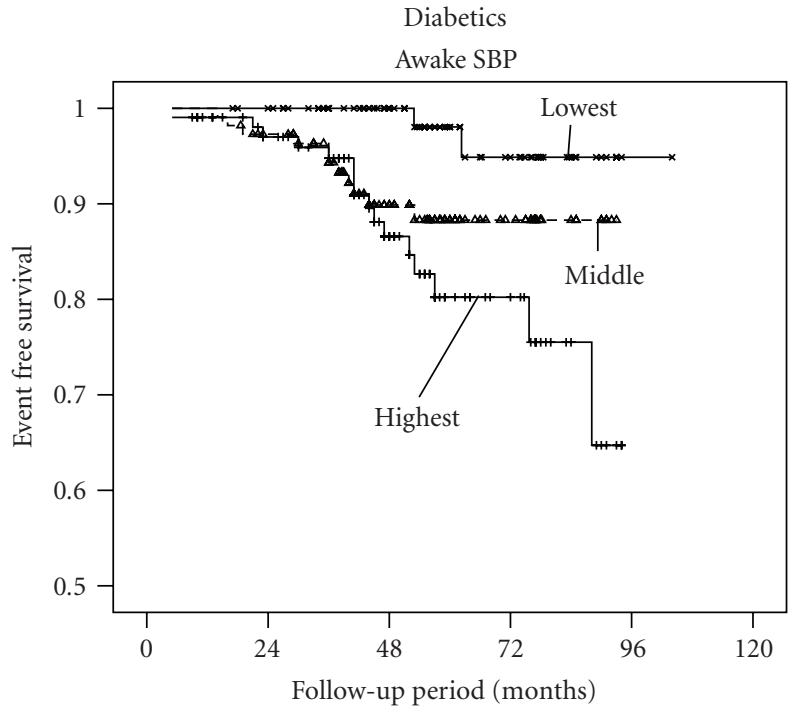

(a)

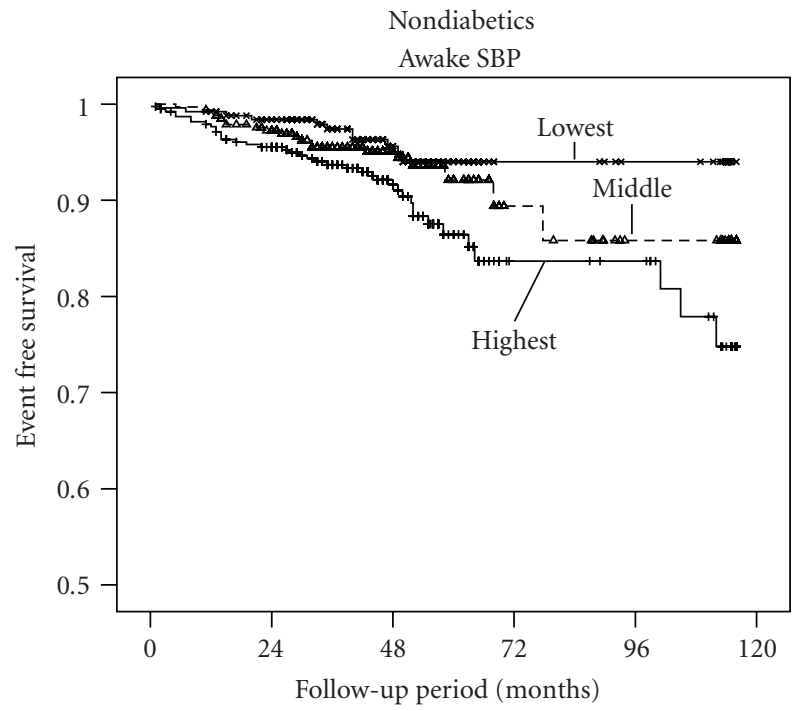

(c)

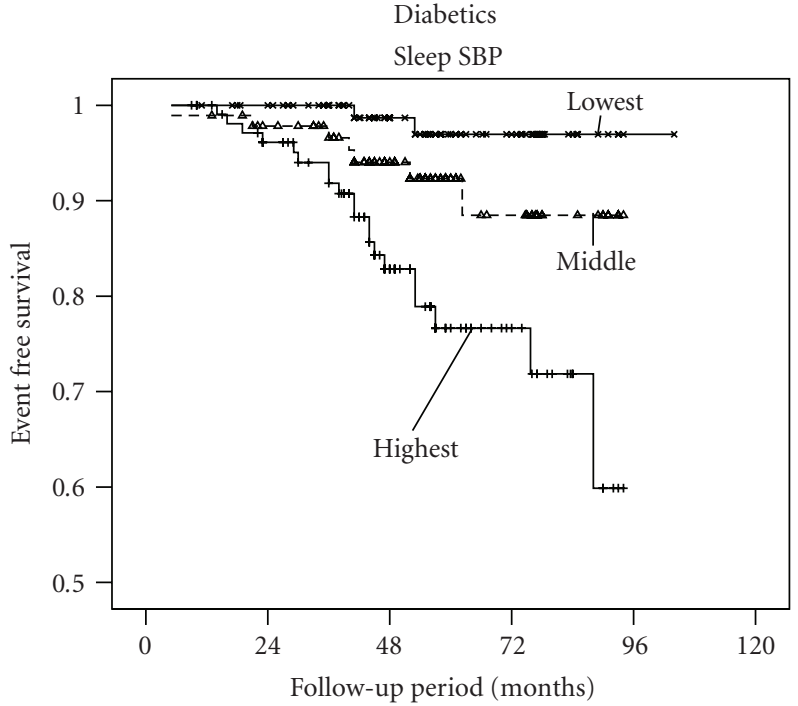

(b)

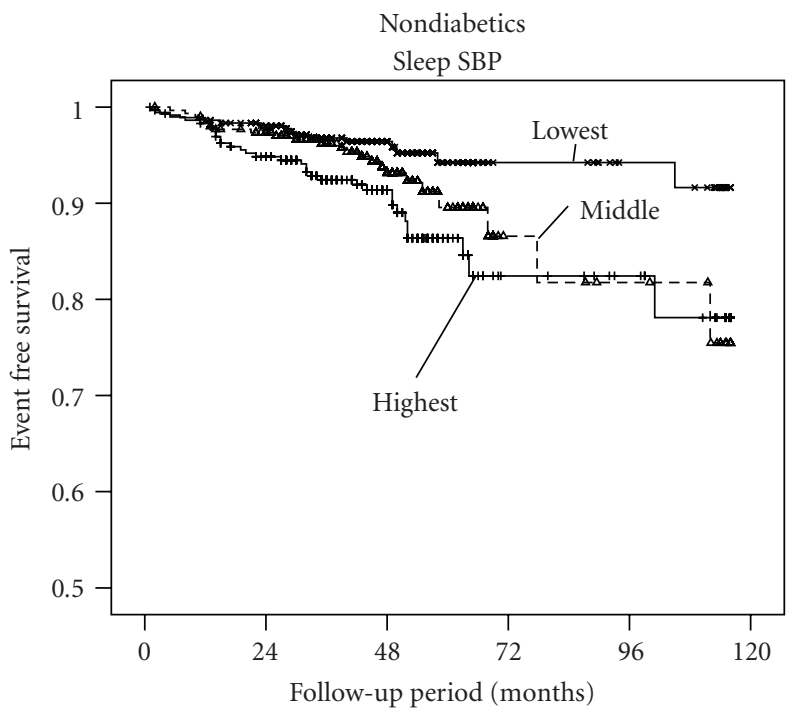

(d)

FIGURE 3: Event-free survival Kaplan-Meier curves for three categories of awake and sleep SBP. The log-rank statistic between the highestand lowest-awake SBP is $11.2(P=.001)$ for the diabetes and $8.4(P=.004)$ for the non-diabetes group, while that between the middle- and lowest-awake SBP is $4.5(P=.03)$ for the diabetes and $1.0(P=.32)$ for the non-diabetes group. The log-rank statistic between the highestand middle-awake SBP is $1.8(P=.19)$ for the diabetes and $4.0(P=.046)$ for the non-diabetes group. The log-rank statistic between the highest- and lowest-sleep SBP is $16.3(P<.001)$ for the diabetes and $11.3(P=.001)$ for the non-diabetes group, and that between the middle- and lowest-sleep SBP is $3.3(P=.07)$ for the diabetes and $3.8(P=.05)$ for the non-diabetes group. The log-rank statistic between the highest- and lowest-sleep SBP is $6.4(P=.01)$ for the diabetes and $1.9(P=.17)$ for the non-diabetes group. SBP indicates systolic blood pressure (adopted from [10]).

\section{Ambulatory Blood Pressure Monitoring in the Obese}

There has been conflicting evidence that obesity or overweight is associated with abnormal circadian rhythm of BP. Kotsis et al. demonstrated that normal nocturnal BP reduction was significantly decreased from normal weight to obese in 3,216 untreated hypertensive patients. The prevalence of nondippers was much greater in the obese than in the normal weight group $(71.4 \%$ versus $41.1 \%$ in the normotensive and $72.7 \%$ versus $61.5 \%$ in the hypertensive subsets) [23]. In the Oman Family Study $(n=1,124)$, BMI was reported to be significantly associated with a nocturnal dipping pattern $(r=-0.23)$ in a multivariate model among patients with metabolic syndrome [24]. In a data from the Spanish Society of Hypertension registry, obesity was one of the determinants of non-dipper pattern [2]. In contrast, Diamantopoulos et al. examined 226 (116 male and 110 female) overweight 


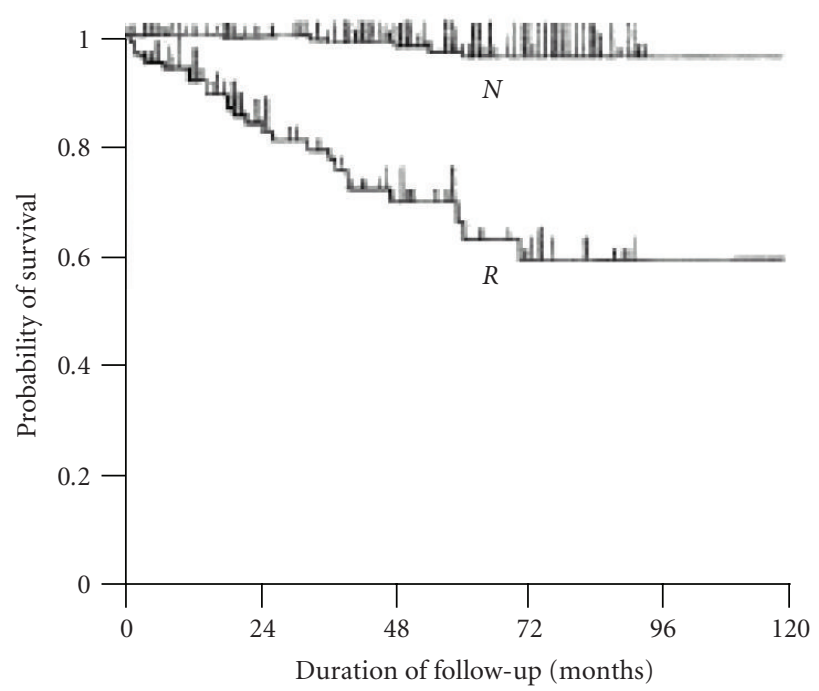

(a)

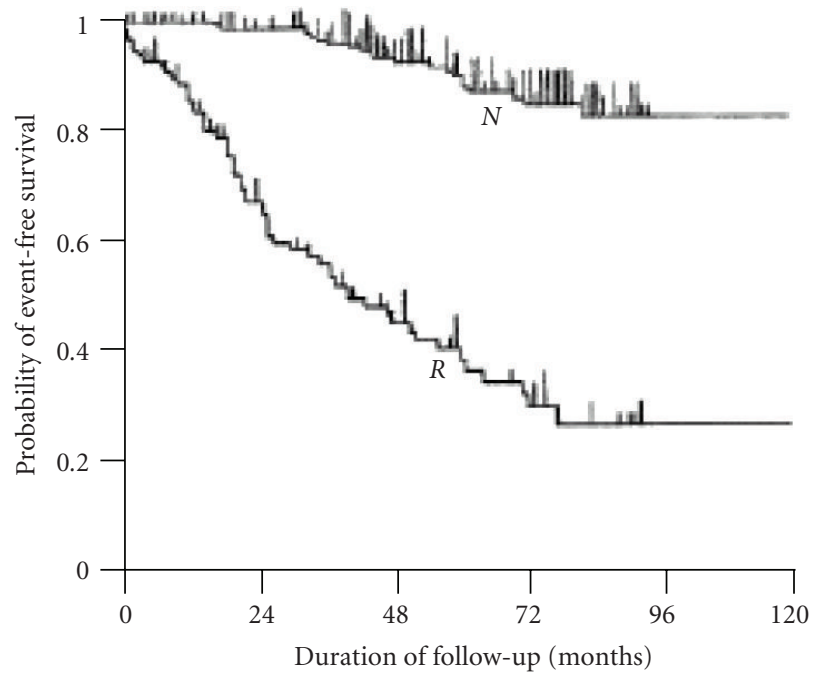

(b)

FIGURE 4: Survival curves (a) and event-free survival curves (b) of diabetic subjects with normal (N) and reversed circadian BP rhythm. The unadjusted relative risk for diabetic subjects with a reversed circadian BP rhythm was 20.6-fold higher than that of subjects with a normal rhythm $(P<.001$; Cox-Mantel's test) for survival curves (a), and 12.9-fold higher than that of subjects with a normal rhythm $(P<.001$; Cox-Mantel's test) for event-free survival curves (b) (adopted from [11]).

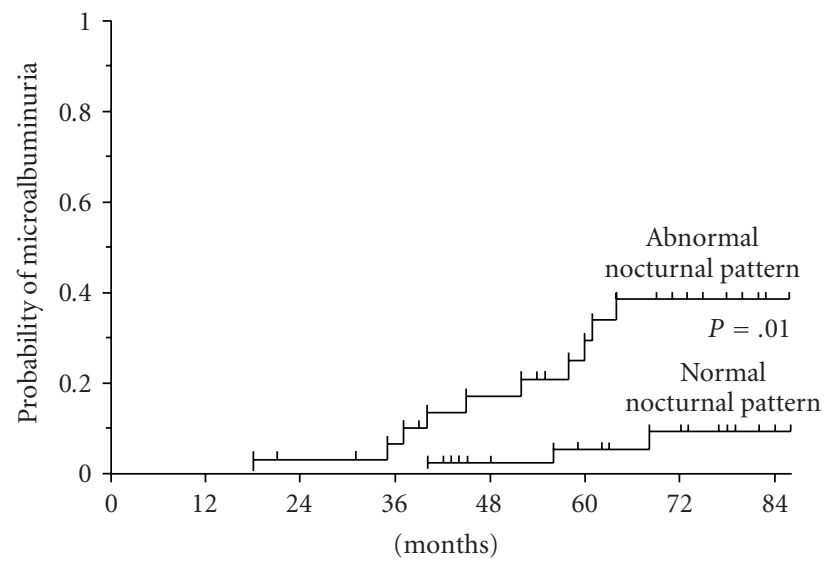

Figure 5: Kaplan-Meier curves showing the probability of microalbuminuria according to the pattern of daytime and nighttime systolic pressure. The probability of microalbuminuria differed significantly between the two groups $(P=.01$ by the log-rank test). The risk of microalbuminuria was $70 \%$ lower in the subjects with a normal nocturnal pattern (adopted from [16]).

and obese subjects $\left(\right.$ BMI $\left.>27 \mathrm{~kg} / \mathrm{m}^{2}\right)$ with newly diagnosed essential hypertension. There were no significant differences in BMI between the dippers and nondippers [25]. Obese men had an increased heart rate and diastolic BP compared to normal and overweight men, but those were similar between the normal and obese women [26]. Such a gender difference could reflect some pathophysiological mechanism of BP and obese status. Because obesity is associated with many other factors, such as diabetes, Metabolic Syndrome (MS), and Sleep apnea syndrome, it is difficult to isolate any single

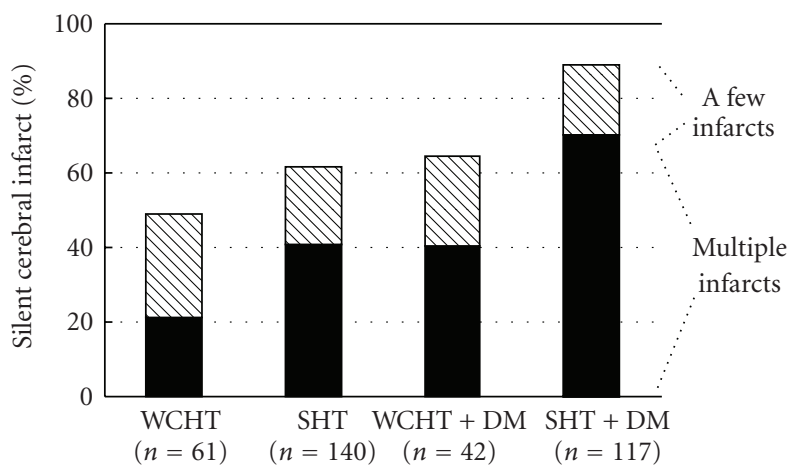

FIgure 6: Prevalence of SCIs detected by brain MRI. Multiple SCIs are defined as $\geq 3$ infarcts per person. The overall probability values for 4-group comparisons were determined by chi-square test as shown in Table 1 (adopted from [17]).

component in human studies. Further studies will be needed to examine the relationship between obesity and dipping patterns.

\section{Metabolic Syndrome and Circadian Rhythm of BP}

There has been accumulating evidence that metabolic syndrome is associated with nondipping of BP. Hermida et al. studied 1770 nondiabetic untreated hypertensive patients, and performed ABPM for 48 hours [27]. They found that the prevalence of a nondipping profile was significantly higher in patients with MS ( $46.1 \%$ versus $37.5 \%$ in patients without MS, $P<.001)$. The single most relevant factor in the 


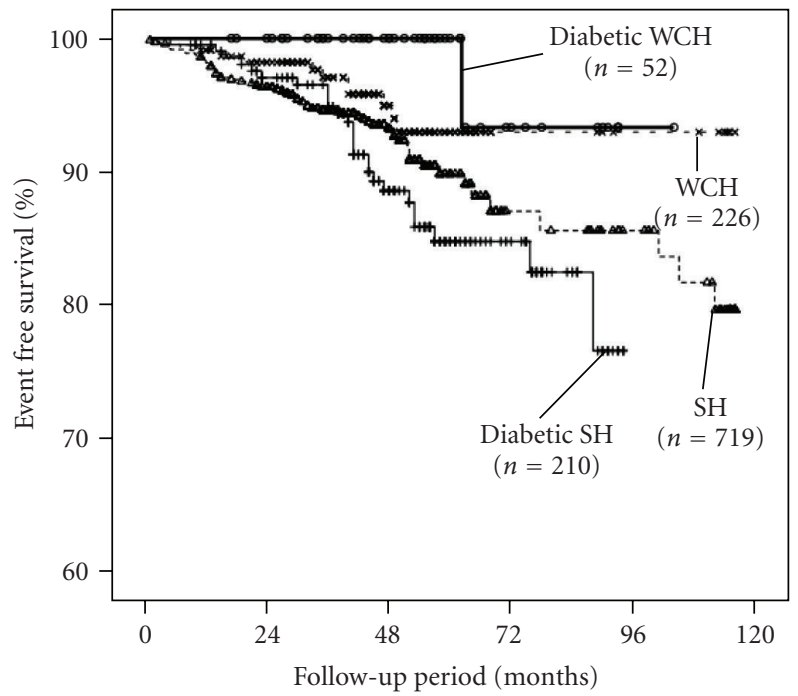

Figure 7: Curves for event-free survival without a cardiovascular event. The overall log-rank statistic for the 4-group comparison was $8.8(P=.032)$. The log-rank statistics for the intergroup comparisons were $4.9(P=.028$; diabetic $\mathrm{SH}$ versus diabetic $\mathrm{WCH})$, $2.08(P=.15$; diabetic $\mathrm{SH}$ versus $\mathrm{SH})$, and $5.1(P=.024$; diabetic $\mathrm{SH}$ versus $\mathrm{WCH})$. However, the value in the diabetic $\mathrm{WCH}$ group was not significantly different from that in the SH group $\left(\chi^{2}=3.0\right.$, $P=.08)$ or WCH group $\left(\chi^{2}=1.2, P=.28\right)$ and that in the $\mathrm{SH}$ group was not significantly different from that in the WCH group $\left(\chi^{2}=2.2, P=.14\right)$. SH, sustained hypertension; $\mathrm{WCH}$, white-coat hypertension (Adopted from [20]).

definition of MS associated with nondipping was elevated waist circumference. There are other reports supporting the relationship between MS and a nondipping pattern [24, 28-30]. Additionally, nondipping status has been shown to be an independent predictor of glucose tolerance and several other metabolic abnormalities [31]. In contrast, other studies reported no difference in the ambulatory BP patterns between patients with and those without MS. With regard to the components of MS, glucose and abdominal obesity were two important components, but the number of components itself was also a determinant of the nondipping pattern. The common pathological states between MS and the nondipping status would be hyperinsulinemia and resultant sympathetic hyperactivity. However, it is not clear which component is the most important factor in determining nondipping status in the MS, because many factors which are related to nondipping patterns, such as abdominal obesity, diabetes, and sleep apnea syndrome, coexist in the same individuals. Further studies will be needed to clarify the relationship between MS and the nondipping pattern.

\section{Conclusion}

In patients with diabetes and obesity, ABPM should be performed at least once for the better risk stratification of hypertension. Early detection of nocturnal hypertension (in its most extreme form, a "riser pattern") is very important for preventing cardiovascular events. ABPM data are also effective to improve adherence to therapy. Further studies will be needed for the proper application of ABPM in such high-risk populations.

\section{References}

[1] W. C. Cushman, G. W. Evans, R. P. Byington et al., "Effects of intensive blood-pressure control in type 2 diabetes mellitus," The New England Journal of Medicine, vol. 362, no. 17, pp. 1575-1585, 2010.

[2] A. de la Sierra, J. Redon, J. R. Banegas et al., "Prevalence and factors associated with circadian blood pressure patterns in hypertensive patients," Hypertension, vol. 53, no. 3, pp. 466472, 2009.

[3] P. Home, "Global guideline for type 2 diabetes: recommendations for standard, comprehensive, and minimal care," Diabetic Medicine, vol. 23, no. 6, pp. 579-593, 2006.

[4] T. Ogihara, K. Kikuchi, H. Matsuoka et al., "The Japanese Society of Hypertension Guidelines for the Management of Hypertension (JSH 2009)," Hypertension Research, vol. 32, no. 1, pp. 3-107, 2009.

[5] American Diabetes Association, "Diagnosis and classification of diabetes mellitus," Diabetes Care, vol. 34, supplement 1, pp. S62-S69, 2010.

[6] S. Lehto, T. Rönnemaa, K. Pyörälä, and M. Laakso, "Predictors of stroke in middle-aged patients with non-insulin-dependent diabetes," Stroke, vol. 27, no. 1, pp. 63-68, 1996.

[7] R. Turner, R. Holman, I. Stratton et al., "Tight blood pressure control and risk of macrovascular and microvascular complications in type 2 diabetes: UKPDS 38," British Medical Journal, vol. 317, no. 7160, pp. 703-713, 1998.

[8] I. M. Stratton, A. I. Adler, H. A. W. Neil et al., "Association of glycaemia with macrovascular and microvascular complications of type 2 diabetes (UKPDS 35): prospective observational study," British Medical Journal, vol. 321, no. 7258, pp. 405-412, 2000.

[9] K. Eguchi, J. Ishikawa, S. Hoshide, S. Ishikawa, K. Shimada, and K. Kario, "Impact of blood pressure vs. glycemic factors on target organ damage in patients with type 2 diabetes mellitus," Journal of Clinical Hypertension, vol. 8, no. 6, pp. 404-410, 2006.

[10] K. Eguchi, T. G. Pickering, S. Hoshide et al., "Ambulatory blood pressure is a better marker than clinic blood pressure in predicting cardiovascular events in patients with/without type 2 diabetes," American Journal of Hypertension, vol. 21, no. 4, pp. 443-450, 2008.

[11] S. Nakano, K. Konishi, K. Furuya et al., "A prognostic role of mean 24-h pulse pressure level for cardiovascular events in type 2 diabetic subjects under 60 years of age," Diabetes Care, vol. 28, no. 1, pp. 95-100, 2005.

[12] K. Eguchi, J. Ishikawa, S. Hoshide, T. G. Pickering, K. Shimada, and K. Kario, "Masked hypertension in diabetes mellitus: a potential risk," Journal of Clinical Hypertension, vol. 9, no. 8, pp. 601-607, 2007.

[13] K. Eguchi, T. G. Pickering, and K. Kario, "Why is blood pressure so hard to control in patients with type 2 diabetes?" Journal of the Cardiometabolic Syndrome, vol. 2, no. 2, pp. 114118, 2007.

[14] C. Cuspidi, S. Meani, L. Lonati et al., "Short-term reproducibility of a non-dipping pattern in type 2 diabetic hypertensive patients," Journal of Hypertension, vol. 24, no. 4, pp. 647-653, 2006. 
[15] S. Nakano, M. Fukuda, F. Hotta et al., "Reversed circadian blood pressure rhythm is associated with occurrences of both fatal and nonfatal vascular events in NIDDM subjects," Diabetes, vol. 47, no. 9, pp. 1501-1506, 1998.

[16] E. Lurbe, J. Redon, A. Kesani et al., "Increase in nocturnal blood pressure and progression to microalbuminuria in type 1 diabetes," The New England Journal of Medicine, vol. 347, no. 11, pp. 797-805, 2002.

[17] K. Kario, T. G. Pickering, T. Matsuo, S. Hoshide, J. E. Schwartz, and K. Shimada, "Stroke prognosis and abnormal nocturnal blood pressure falls in older hypertensives," Hypertension, vol. 38, no. 4, pp. 852-857, 2001.

[18] K. Eguchi, K. Kario, and K. Shimada, "Greater impact of coexistence of hypertension and diabetes on silent cerebral infarcts," Stroke, vol. 34, no. 10, pp. 2471-2474, 2003.

[19] K. Kario and T. G. Pickering, "White-coat hypertension or white-coat hypertension syndrome: which is accompanied by target organ damage?" Archives of Internal Medicine, vol. 160, no. 22, pp. 3497-3498, 2000.

[20] K. Eguchi, S. Hoshide, J. Ishikawa et al., "Cardiovascular prognosis of sustained and white-coat hypertension in patients with type 2 diabetes mellitus," Blood Pressure Monitoring, vol. 13, no. 1, pp. 15-20, 2008.

[21] C. K. Kramer, C. B. Leitão, L. H. Canani, and J. L. Gross, "Impact of white-coat hypertension on microvascular complications in type 2 diabetes," Diabetes Care, vol. 31, no. 12, pp. 2233-2237, 2008.

[22] C. B. Leitão, L. H. Canani, C. K. Kramer, J. C. Boza, A. F. Pinotti, and J. L. Gross, "Masked hypertension, urinary albumin excretion rate, and echocardiographic parameters in putatively normotensive type 2 diabetic patients," Diabetes Care, vol. 30, no. 5, pp. 1255-1260, 2007.

[23] V. Kotsis, S. Stabouli, M. Bouldin, A. Low, S. Toumanidis, and N. Zakopoulos, "Impact of obesity on 24-hour ambulatory blood pressure and hypertension," Hypertension, vol. 45, no. 4, pp. 602-607, 2005.

[24] M. O. Hassan, D. Jaju, S. Albarwani et al., "Non-dipping blood pressure in the metabolic syndrome among Arabs of the Oman family study," Obesity, vol. 15, no. 10, pp. 2445-2453, 2007.

[25] E. J. Diamantopoulos, E. Andreadis, G. Tsourous et al., "Insulin resistance and blood pressure circadian variation in an obese hypertensive population," Clinical and Experimental Hypertension, vol. 28, no. 7, pp. 625-630, 2006.

[26] A. Kagan, H. Faibel, G. Ben-Arie, Z. Granevitze, and J. Rapoport, "Gender differences in ambulatory blood pressure monitoring profile in obese, overweight and normal subjects," Journal of Human Hypertension, vol. 21, no. 2, pp. 128-134, 2007.

[27] R. C. Hermida, L. Chayán, D. E. Ayala et al., "Association of metabolic syndrome and blood pressure nondipping profile in untreated hypertension," American Journal of Hypertension, vol. 22, no. 3, pp. 307-313, 2009.

[28] Z. Tartan, H. Uyarel, H. Kasikcioglu et al., "Metabolic syndrome as a predictor of non-dipping hypertension," Tohoku Journal of Experimental Medicine, vol. 210, no. 1, pp. 57-66, 2006.

[29] G. P. Vyssoulis, E. A. Karpanou, S. M. G. Kyvelou et al., "Nocturnal blood pressure fall and metabolic syndrome score in hypertensive patients," Blood Pressure Monitoring, vol. 12, no. 6, pp. 351-356, 2007.
[30] D. E. Ayala, R. C. Hermida, L. Chayán, A. Mojón, M. J. Fontao, and J. R. Fernández, "Circadian pattern of ambulatory blood pressure in untreated hypertensive tatients with and without metabolic syndrome," Chronobiology International, vol. 26, no. 6, pp. 1189-1205, 2009.

[31] O. Ukkola, R. L. Vasunta, and A. Y. Kesäniemi, "Non-dipping pattern in ambulatory blood pressure monitoring is associated with metabolic abnormalities in a random sample of middleaged subjects," Hypertension Research, vol. 32, no. 11, pp. 1022-1027, 2009. 


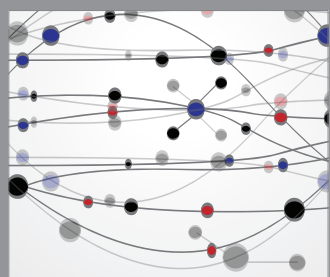

The Scientific World Journal
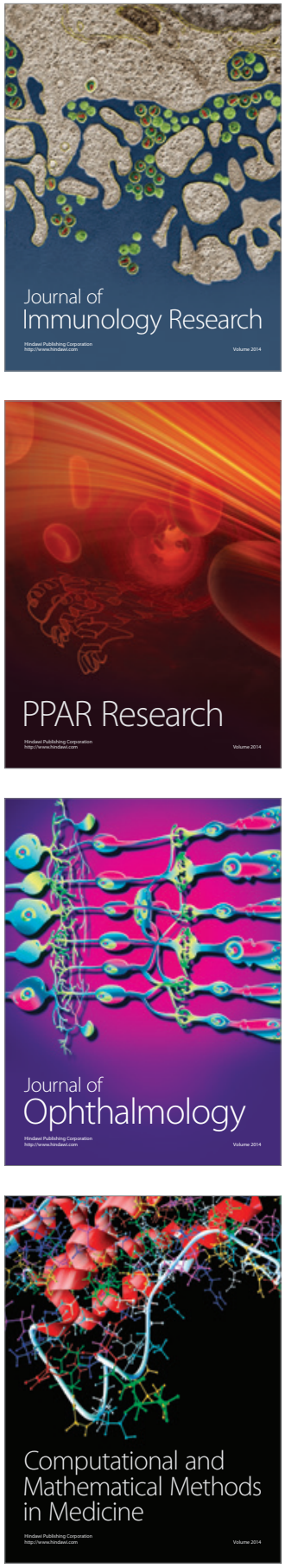

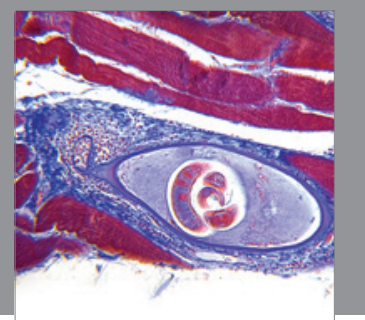

Gastroenterology

Research and Practice
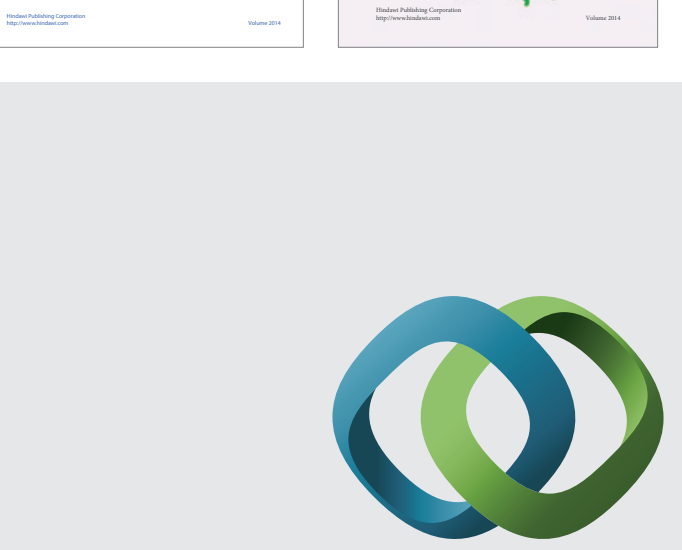

\section{Hindawi}

Submit your manuscripts at

http://www.hindawi.com
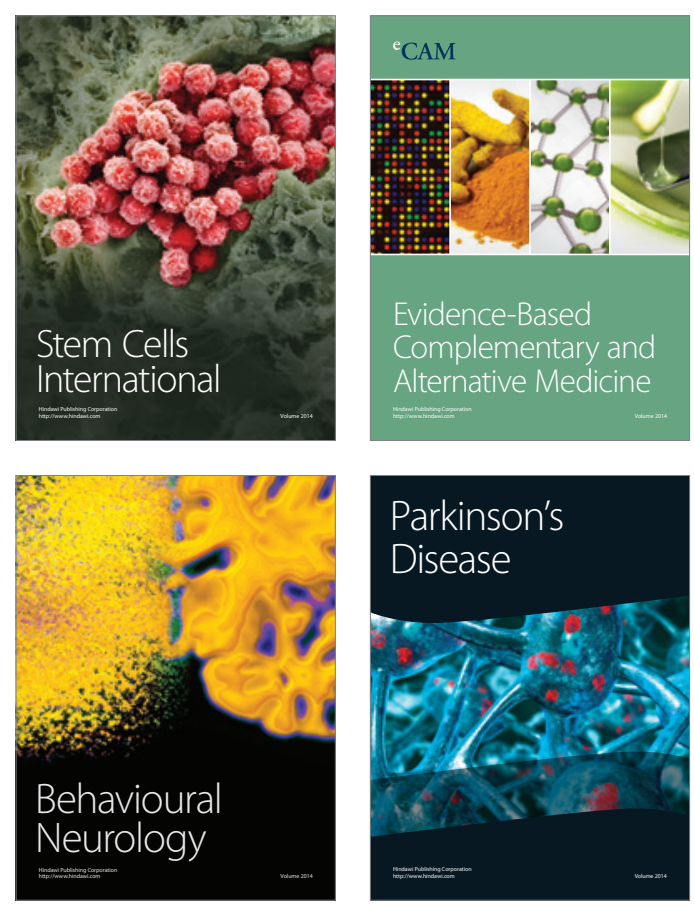

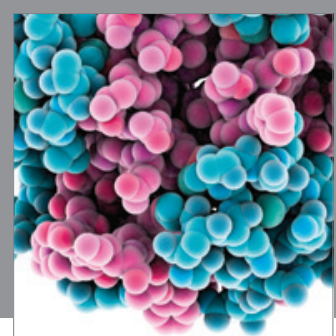

Journal of
Diabetes Research

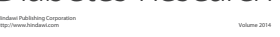

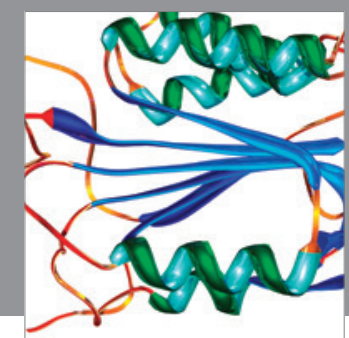

Disease Markers
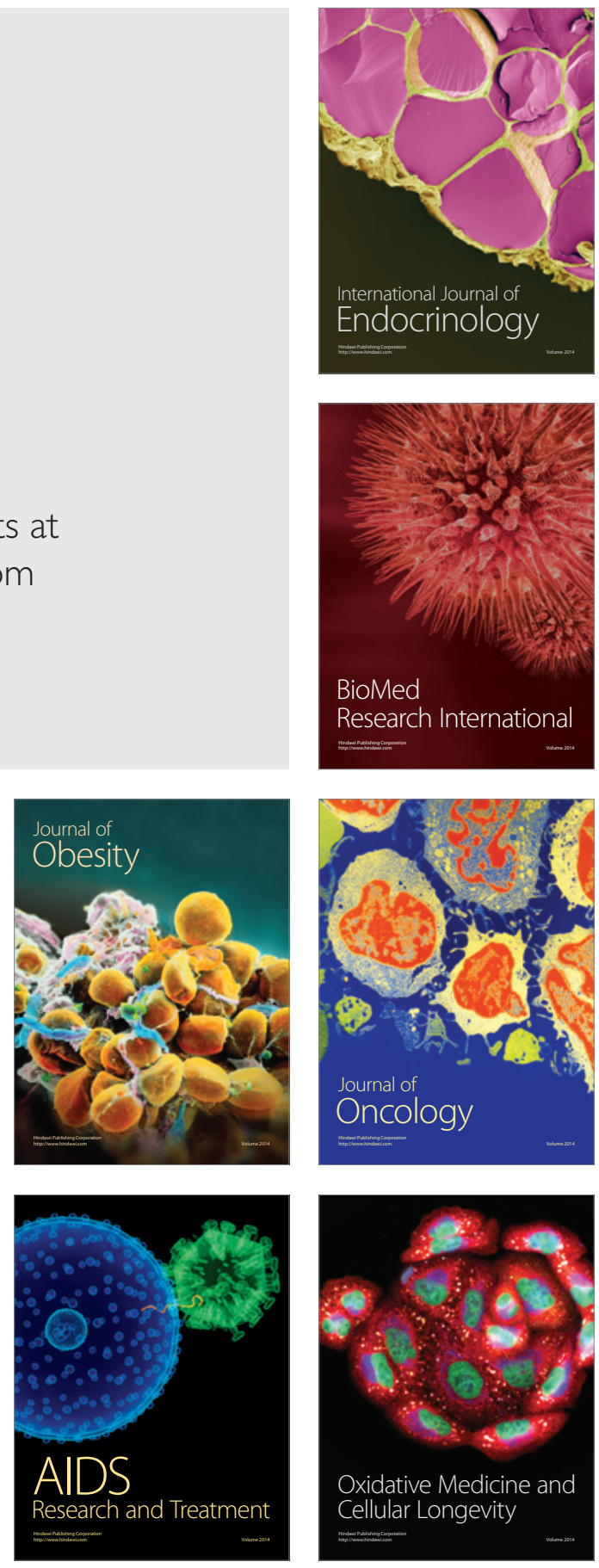